\title{
Wofür braucht die Medizinethik empirische Methoden?
}

\section{Eine normativ-ethische Untersuchung}

\author{
Marcus Düwell
}

Online publiziert: 27. August 2009

(C) Die Autor(en) 2009. Dieser Artikel ist auf Springerlink.com mit Open Access verfügbar.

Zusammenfassung Der Einsatz empirischer Forschungsmethoden in der Medizinethik hat zu Forderungen nach einem gewandelten Selbstverständnis der Medizinethik geführt, die sich mehr als eine integrierte Disziplin aus Sozialwissenschaften und Ethik verstehen solle. Dagegen wird hier die These vertreten, dass über Sinn und Unsinn des Einsatzes empirischer Methoden zunächst eine moralphilosophische Diskussion erforderlich ist. Medizinethiker müssen ausweisen können, welche empirischen Forschungsresultate zur Beantwortung normativer Fragen erforderlich sind. Ein solcher Ausweis beruht seinerseits jedoch auf normativen Annahmen, die ihrerseits moralphilosophischer Legitimation bedürfen. Der Beitrag untersucht daher den Einsatz empirischer Methoden in der Medizinethik im Hinblick auf Verhältnis von ethischem Grundlagendiskurs und Methodologie der angewandten Ethik. Abschließend wird gefragt, ob nicht ein Großteil von empirischen Studien in der Medizinethik aus forschungsethischen Gründen problematisch ist.

Schlüsselwörter Empirische Ethik · Sozialwissenschaften · Interdisziplinarität · Ethische Methodologie $\cdot$ Metaethik

\section{What does medical ethics need empirical methods for?}

An inquiry in normative ethics

Abstract Definition of the problem The use of empirical methods in medical ethics and bioethics has generated proposals for reconceptualizing medical ethics as a discipline that integrates ethics and social science. Arguments The position taken in the present paper, by contrast, is that what is needed, in the first place, is a moral-philosophical evaluation of when it makes sense to employ empirical methods. Medical ethicists must demonstrate what empirical research is necessary for answering normative questions. Any such justification will, however, necessarily rely on normative assumptions that, for their part,

M. Düwell $(\bowtie)$

Ethics Institute, Department for Philosophy, Utrecht University

Heidelberglaan 8, 3584 CS Utrecht, The Netherlands

E-Mail: m.duwell@uu.nl 
require moral-philosophical justification. The paper examines the use of empirical methods in medical ethics in terms of the appropriate relationship between the methodology of applied ethics and debates over the foundations of ethics. The paper concludes by raising the question of whether it is not the case that most empirical studies in medical ethics are of dubious merit, by the accepted standards of research ethics.

Keywords Empirical ethics · Social sciences · Interdisciplinarity · Ethical methodology · Meta-ethics

\section{Einleitung}

Die Verwendung von sozialwissenschaftlichen Methoden in der Medizinethik führt zu einer Reihe von methodischen Fragen: Kann es eine „empirische Ethik“ geben? Was genau kann die normativ-ethische Diskussion von empirischen Untersuchungen erwarten? Wie verhält sich der Rückgriff auf empirische Untersuchungen zum Sein-Sollens-Problem und dem „,naturalistischen Fehlschluss“? Aber auch zu praktischen Fragen: Wie steht es um die sozialwissenschaftliche Kompetenz derer, die diese Untersuchungen durchführen? Wie kann ein interdisziplinärer Dialog zwischen Ethik und Sozialwissenschaften gelingen? (vgl. $[2-6,8,9,11,13,14])$

Im vorliegenden Beitrag soll es um einige dieser Grundfragen zum Einsatz sozialwissenschaftlicher Methoden in der Medizinethik gehen: (1) um eine Diskussion metaethischer Probleme im Verhältnis von Ethik und Sozialwissenschaften, (2) um eine Unterscheidung empirischer Fragen, die für ethische Diskussionen relevant sind, (3) um eine Einordnung dieser Diskussion in grundlegende methodische Überlegungen zur Angewandten Ethik, (4) um Fragen zur Interdisziplinarität und (5) schließlich um forschungsethische Probleme empirischer Ethikforschung.

\section{Metaethische Voraussetzungen}

Ich gehe davon aus, dass es Aufgabe der Ethik ist, die Gültigkeit moralischer Urteile kritisch zu reflektieren und zu prüfen; Aufgabe der Medizinethik ist es, dies im Hinblick auf den Handlungsbereich der Medizin zu tun. Wenn sozialempirische Methoden in der Medizinethik verwendet werden, so kann man erwarten, dass die metaethischen Voraussetzungen dieser Verwendung im Hinblick auf die genannte Aufgabe der Ethik rekonstruiert und legitimiert werden können. Es ist schließlich nicht selbstverständlich, dass Untersuchungen zu bestehenden sozialen Praktiken, Überzeugungen von beteiligten Akteuren etc. Aufschluss darüber geben, wie wir handeln sollen. Es müsste gezeigt werden, warum empirische Untersuchungen zur Begründung moralischer Urteile erforderlich sind. Um dies zeigen zu können, scheint zunächst eine adäquate Bestimmung des Verhältnisses von Fakten, Werten und Normen wesentlich zu sein.

Nun könnte man denken, dass sich eine empirisch arbeitende Ethik metaethisch nur dann rechtfertigen ließe, wenn man zeigen kann, dass die Richtigkeit moralischer Überzeugungen von den faktischen Wertüberzeugungen der Menschen abhängig wäre. Die metaethische Herausforderung bestünde dann entweder darin, eine Dualität von Werten und Fakten zurückzuweisen oder zu zeigen, dass moralische Intuitionen einen unhintergehbaren Ausgangspunkt moralischer Argumentation bilden müssen. Die erste Strategie würde dafür argumentieren, dass es legitim ist, in ethischen Debatten auf Fakten zurückzugreifen, falls es 
keinen garstigen Graben zwischen Fakten und Werten gibt. Diese Argumentationsstrategie wird dann entweder zeigen, dass sich eine naturalistische Interpretation moralischer Fragen verteidigen lässt oder dass uns auch Fakten nicht unabhängig von Wertannahmen zugänglich sind. Die zweite Strategie wird dagegen versuchen einen Intuitionismus zu verteidigen, der das Interesse der Ethik an den Intuitionen der Menschen plausibel macht; sozialempirische Untersuchungen wären dann gewissermaßen der Versuch, methodisch kontrollierten Zugang zu verbreiteten Intuitionen von Menschen zu erhalten.

Nun hat die Diskussion um empirische Ethik sich wesentlich mit der Zurückweisung eines vermeintlichen Dualismus von Fakten und Werten beschäftigt ([22], dazu auch: [11, 21]). Bevor man diesen Dualismus zurückweist, müsste jedoch zunächst gefragt werden, wie dieser Dualismus zu verstehen ist und warum diese Diskussion für das Thema überhaupt relevant ist. So wird etwa ein ontologischer Dualismus von Fakten und Werten zurückgewiesen. Falls diese Zurückweisung erfolgreich ist, so hat man damit jedoch lediglich gezeigt, dass es keine wertfrei zugänglichen Fakten gibt oder dass Werte keine eigenständige ontologische Sphäre bilden. Nun kann man zumindest aus zwei Gründen bestreiten, dass sich an dieser Frage die empirisch-ethischen Geister scheiden: Zum einen ist ein ontologischer Dualismus in der Ethik nur für wenige Theorien des moralischen Realismus überhaupt relevant (dazu: [23, 24]). Für die meisten gängigen ethischen Ansätze (Utilitarismus, Kantianismus, Reflexionsgleichgewicht und Kontraktualismus) ist die Annahme eines ontologischen Dualismus nicht erforderlich. Allenfalls wird ein epistemologischer Dualismus vertreten, der etwa sagt, dass eine Einsicht in Fakten nicht umittelbar zur Erkenntnis von normativer Richtigkeit führt.

Eine erfolgreiche Zurückweisung des ontologischen Dualismus bedeutet nicht, dass naturalistische Erklärungen der Moral schon legitimiert sind, denn der Verweis darauf, dass Werte keinen eigenständigen ontologischen Status haben, bedeutet noch nicht, dass moralische Fragen durch Verweis auf Faktisches beantwortet werden könnten. Auch wenn wir erkennen, dass unser Zugang zur Wirklichkeit notwendig durch Wertannahmen bestimmt wird, bleibt die Frage bestehen, welche dieser Werte legitimer Weise moralisch richtiges Handeln anleiten sollen. Auch bei nicht-dualistischen Konzeptionen ist die Frage, was moralisch richtig ist, von Fragen der Beschaffenheit der Wirklichkeit oder Fragen danach, wie die Menschen faktisch über moralische Fragen denken, unterschieden. Zur Begründung der Richtigkeit moralischer Normen wird auch der Nicht-Dualist auf normativ-ethische Theorien zurückgreifen müssen. Man kann also fragen, ob die Diskussionen um den ontologischen Dualismus und teils auch um den naturalistischen Fehlschluss überhaupt die relevanten Diskussionen sind, wenn es um Sinn und Unsinn empirischer Methoden in der angewandten Ethik geht. Damit will ich nicht behaupten, dass diese Diskussionen für die Relevanz des Empirischen irrelevant sind, aber es wäre zunächst zu zeigen, welche Bedeutung diese Diskussionen genau haben.

Anders sieht es aus, wenn man eine intuitionistische Theorie vertritt, also moralische Intuitionen von Menschen als Ausgangspunkt moralischer Diskurse ansieht [1]; falls eine solche Theorie gerechtfertigt wäre, hätte dies zweifellos für das Verhältnis von Empirie und Ethik grundlegende Bedeutung. Allerdings kann der Rekurs auf Intuitionen sehr unterschiedliche Funktionen haben: Die zwei extremen Möglichkeiten wären rechtfertigende oder heuristische Funktionen. Vertritt man eine Theorie, nach der moralische Intuitionen als Kriterien richtigen Handelns fungieren, so könnte die Konsequenz sein, dass man sozialempirische Untersuchungen nutzt, um moralische Intuitionen zu erheben. Eine solche Form des Intuitionismus muss allerdings eine Reihe von Fragen beantworten, etwa danach, warum wir davon ausgehen können, dass unsere Intuitionen einen verlässlichen Kompass für moralisch strittige Fragen abgeben. Oder die Frage, wie wir mit gegenstreitigen Intuitionen umgehen 
sollen. Vertritt man hingegen eine Theorie, wonach moralischen Intuitionen eine heuristische Funktion zukommt, so mag es interessant sein, die moralischen Intuitionen zu erheben, doch anschließend ist ein heuristischer Intuitionist mit der Frage konfrontiert, mit welchen Kriterien er die moralische Relevanz der ermittelten Intuitionen dann bewerten will. Das ganze Spektrum der möglichen normativ-ethischen Ansätze kehrt dann zurück. Sofern man den Intuitionismus als theoretischen Rahmen für die Konzeption des Verhältnisses von Ethik und Sozialwissenschaften ansieht, wäre allerdings bereits vorgegeben, was genau die Sozialwissenschaften untersuchen sollten: die moralischen Intuitionen von Menschen.

Der kurze Ausflug in die Metaethik zeigt einen grundsätzlichen Aspekt des Themas: Die Vertretbarkeit einer Einbeziehung empirischer Methoden in die Medizinethik ist nicht unabhängig von metaethischen Annahmen, sondern begründungsbedürftig. Dabei ist eine Zurückweisung eines Fakten-Werte-Dualismus noch keine hinreichende Legitimation für den Rückgriff auf empirische Methoden. Grundsätzlich steht man aber vor der Schwierigkeit, dass diese metaethischen Annahmen philosophisch umstritten sind und wahrscheinlich auch umstritten bleiben. Die Medizinethik wird sich zu dieser Diskussion verhalten müssen.

\section{Empirische Methoden und normative Ethik}

Die Einbeziehung sozialwissenschaftlicher Methoden in die medizinethische Diskussion ist aber auch im Hinblick auf normativ-ethische Theorien nicht neutral. Das zeigt sich bereits daran, welche empirischen Fragen man zu erheben denkt. Diese Behauptung soll anhand einiger möglicher empirischer Fragestellungen verdeutlicht werden. Ich wähle exemplarisch den Kontext der Sterbehilfe, wobei ich den Begriff bewusst weit halte und mich nicht auf bestimmte Formen der Sterbehilfe (aktiv, passiv, indirekt, Tötung auf Verlangen) beschränke. Die Medizinethik könnte an sozialwissenschaftlichen Forschungen zu folgenden Fragen interessiert sein:

(a) Wie sieht die Praxis von Entscheidungen am Lebensende (in bestimmten Ländern, Krankenhäusern etc.) aus?

(b) Welche Prognosen haben verschiedene Gruppen bezüglich der Praxis sofern Regelungen der Sterbhilfe verändert werden? Welche alternativen Regelungen sehen sie als denkbar an?

(c) Wie wird die bestehende Praxis von Entscheidungen am Lebensende wahrgenommen und bewertet?

(d) Welche Präferenzen haben die Öffentlichkeit, bestimmte Berufs-, Alters- oder soziale Gruppen bezüglich möglicher Regelungen der Sterbehilfe?

(e) Welche Regelungen der Sterbehilfe werden als moralisch legitim angesehen?

(f) Welche Argumente haben die verschiedenen Gruppen für ihr moralisches Urteil?

Die Liste ist nicht vollständig, aber für den vorliegenden Zweck ausreichend. Frage (a) richtet sich darauf, Einblick darin zu erhalten, wie eine bestehende Praxis faktisch aussieht. Solche Formen empirischer Untersuchungen sind für die Ethik wichtig, insofern eine ethische Beurteilung Wissen über eine bestimmte Praxis voraussetzt und insofern dieses Wissen nur durch Befragung der Betroffenen zu gewinnen ist. Für alle Positionen zur Sterbehilfe etwa, die auf den Schutz der Selbstbestimmung der Betroffenen abheben, wird die entscheidende Frage sein, inwiefern Sterbehilfe-Entscheidungen tatsächlich selbstbestimmt getroffen werden. Zur Beantwortung solcher Fragen nach der bestehenden Praxis sind nur Forschungsmethoden erforderlich, die darauf abzielen, das Wissen von Beteiligten in deren 
Eigenschaft als intime Kenner einer bestimmten Praxis zu erheben; es geht dann nicht um die Wertüberzeugungen dieser Personengruppen.

Frage (b) ist eine prognostische Frage, die ebenfalls darauf abzielt, Kenntnis von Menschen zu erheben, die eine Praxis besonders gut kennen. Das kann etwa relevant sein, wenn slippery slope oder Missbrauch befürchtet wird. Ähnliches gilt für die Erhebung möglicher Regelungsalternativen. Man greift dann auf Kenntnisse von Ärzten, Pflegenden, Vormundschaftsrichtern oder auch Angehörigen zurück, um herauszufinden, wie sich Änderungen der Regelungen aus ihrer Perspektive vermutlich auswirken werden. Auch in dieser Hinsicht werden die Befragten allein im Blick auf ihre besondere Kenntnis des Gegenstandsbereichs ausgewählt.

Frage (c) zielt dagegen darauf, Wertungen von Beteiligten zu erheben. Dies kann etwa relevant sein, wenn es darum geht, die Rolle von Ärzten und Pflegenden zu erheben, die an Entscheidungen am Lebensende direkt beteiligt sind. Ebenso könnte es relevant sein, die Wertungen breiter Bevölkerungsgruppen zum Thema zu erheben, insofern im Falle von Entscheidungen am Lebensende jeder ein potentiell Betroffener ist. Aus ethischer Perspektive wäre allerdings zu begründen, warum man genau wissen muss, wie verschiedene Gruppen die Praxis bewerten. Strittig ist doch, ob eine Praxis wirklich moralisch fragwürdig ist und nicht, wie bestimmte Gruppen darüber denken. Für eine empirische Erhebung der Bewertung von betroffenen Gruppen wäre also eine Rechtfertigung erforderlich, die abhängig von der unterstellten normativ-ethischen Theorie unterschiedlich ausfallen kann.

$\mathrm{Zu}$ berücksichtigen ist allerdings die Besonderheit der Sterbehilfe, bei der jeder ein potentiell Betroffener und somit auch mögliches Objekt der Befragung ist. Das stellt sich anders dar, wenn es etwa um Fragen der Behandlung sehr spezifischer Krankheiten geht, die nur bei besonderen Zielgruppen auftreten. Hier kann es Gründe geben, den Wertannahmen dieser Gruppen ein besonderes Gewicht beizumessen. Etwa im Rahmen einer Theorie von Grundbedürfnissen oder einer Menschenrechtstheorie wäre es denkbar, dass man erst die spezifischen Bedürfnisse von besonders verletzlichen Gruppen erheben muss, deren Bedürfnisse nicht ohne Weiteres bekannt sind.

Frage (d) fragt nach den Präferenzen für eine Regelung der Sterbehilfe, also danach, welche Angebote Einzelne (oder spezifische Gruppen) in Anspruch nehmen wollen. Diese Frage ist für eine ethische Untersuchung lediglich dann relevant, wenn gemäß der unterstellten ethischen Theorie zur Formulierung von Regelungen der Sterbehilfe die Präferenzen der Betroffenen ausschlaggebend sein sollten. Aus der Perspektive von Theorien, die bestimmte Formen der Sterbehilfe aus grundsätzlichen Erwägungen als unvertretbar ansehen, ist es also nicht notwendig, die Präferenzen der Öffentlichkeit oder spezifischer Gruppen zu erheben. Aber auch für viele liberale Theorien ist die Kenntnis von Präferenzen nicht wirklich erforderlich, um ein ethisches Urteil zu bilden. Wenn etwa die normative Ausgangsposition lautet, dass es moralisch geboten ist, alle Formen der Sterbehilfe zu ermöglichen, so wäre es unwesentlich zu wissen, welche Präferenzen die Bürger faktisch haben. Der Liberale müsste sich unabhängig von den faktischen Präferenzen der Bürger verpflichtet sehen, die entsprechenden Angebote zur Verfügung zu stellen, auch wenn manche nur selten genutzt werden. Wesentlich wäre das Erheben der Präferenzen jedoch für solche utilitaristische Theorien, die moralische Richtigkeit von der Erfüllung möglichst vieler Präferenzen abhängig machen. Eine solche Theorie ist ultimativ davon abhängig, faktische Präferenzen zu kennen. Allerdings könnte man fragen, ob empirische Sozialforschung dazu das geeignete Mittel ist oder man nicht lieber Mehrheitsentscheidungen herbeiführt.

Während Frage (d) darauf abzielt, die Präferenzen von Einzelnen oder Gruppen zu erheben, will Frage (e) nicht wissen, was die Befragten für sich selbst wünschen, sondern welche Regelungen sie im Allgemeinen für moralisch vertretbar ansehen. Nun wäre die Frage, 
warum die Tatsache für ein ethisches Urteil relevant sein sollte, dass eine Mehrheit oder eine bestimmte Gruppe etwas als moralisch vertretbar oder unvertretbar ansieht. Ob es für eine bestimmte Regelung gute oder schlechte Argumente gibt, hängt schließlich nicht davon ab, was eine Mehrheit für moralisch ansieht. Ein denkbares Argument ließe sich allenfalls in der Form konstruieren, dass es möglicherweise nicht entscheidbar ist, welche Regelung moralisch besser ist (das müsste dann zunächst argumentativ gezeigt werden). In diesem Fall könnte man argumentieren, dass es in einer Demokratie moralisch vorzuziehen ist, dasjenige zu tun, was eine Mehrheit als moralisch besser ansieht. Dieses Argument würde dann aber nicht begründen, dass es geboten ist, die moralischen Überzeugungen von Gruppen zu erheben, sondern dafür sprechen, demokratisch legitimierte Entscheidungsprozesse herbeizuführen. Im Rahmen der skizzierten Argumentation hätte die Ethik jedenfalls keine eigenen Argumente zu dieser demokratischen Entscheidung beizusteuern, die darüber hinausgehen zu zeigen, dass es eine Art moralische Pattsituation gibt.

Der letzte Fragetyp (f) zielt nicht darauf ab zu erheben, welche moralischen Positionen Menschen haben, sondern welche Argumente sie für diese Positionen haben. Die Erhebung dieser Frage könnte zunächst Erstaunen hervorrufen. Warum sollte eine solche Erhebung Argumente zu Tage fördern, die bislang in der ethischen Literatur noch nicht aufgetaucht sind? Kann sich der Ethiker die Argumente nicht selbst überlegen oder zunächst einmal die Forschungsliteratur konsultieren? Man wird in der Tat von jeder ethischen Studie erwarten können, dass dies zunächst geschieht. Gleichwohl kann es sein, dass in empirischen Untersuchungen Argumente zu finden sind, die bislang nicht oder kaum berücksichtigt wurden. Solche Argumente werden allenfalls durch qualitative empirische Studien zu ermitteln sein. Es könnte sein, dass Philosophen oder Mediziner, die in einem bestimmten akademischen Setting arbeiten, einfach keinen Blick für bestimmte Argumente haben.

Die kurze Übersicht über mögliche Fragestellungen, ist alles andere als vollständig. Es ging auch mehr darum, grundsätzlich anzudeuten, welche Fragen vorgängig beantwortet werden müssen, bevor es entscheidbar ist, in welcher Hinsicht ein Rekurs auf sozialempirische Studien in der Medizinethik sinnvoll ist. Der Grundgedanke lässt sich also wie folgt zusammenfassen: Von verschiedenen normativ-ethischen Positionen her werden sich bestimmte empirische Fragen ergeben und andere nicht. Bisweilen lassen sich diese empirischen Fragen durch Literaturstudium beantworten und bisweilen wird es erforderlich sein, diese empirische Forschung erst durchzuführen. Wir haben gesehen, dass die Relevanz einiger Fragestellungen (etwa nach der bestehenden Praxis oder den in der Öffentlichkeit faktisch vertretenen moralischen Argumenten) weit weniger von normativ-ethischen Vorannahmen abhängig ist, als andere Fragen. Die Bedeutung mancher empirischer Fragestellungen lässt sich nur im Rahmen spezifisch normativ-ethischer Theorien rekonstruieren.

\section{Zur Methodik angewandter Ethik}

Es ergibt sich also die Notwendigkeit, sozialempirische Forschung im Kontext medizinethischer Fragestellungen normativ-ethisch zu legitimieren; eine völlige andere Frage ist es natürlich, ob bestimmte Forschungen medizinsoziologisch sinnvoll sind oder nicht. Im Rahmen der Medizinethik muss hingegen zunächst gezeigt werden, warum spezifische empirische Fragestellungen für die Beantwortung normativ-ethischer Fragen erforderlich sind.

Häufig wird das Problem dadurch gelöst, dass man sich auf Theorieformen bezieht, die sich vermeintlich dem Unterschied normativer und empirischer Theoriebildung entziehen. Dies behaupten etwa pragmatistische Autoren [10] oder Vertreter einer sog. ,integrativen empirischen Ethik“ ([16-18, 25]; dazu auch: [19, 20]). Selbst wenn der Pragmatismus 
jedoch zeigen kann, dass eine dualistische Ontologie unzutreffend ist, bedeutet dies noch nicht, dass die Frage nach gültigen Handlungsnormen und die Frage nach einer zutreffenden Beschreibung der Wirklichkeit nicht unterscheidbar sind. Es sind noch stets drei unterschiedliche Dinge zu behaupten, (a) dass jemand einen anderen getötet hat, (b) dass diese Tötung moralisch unvertretbar ist und dass (c) ein Dritter der Meinung ist, dass diese Tötung moralisch unvertretbar ist. Und die entsprechenden drei Behauptungen sind nicht allein unterscheidbar, sondern es sind zudem unterschiedliche Argumente erforderlich, um eine der drei Behauptungen zu begründen. Das bedeutet jedoch auch, dass die Begründung der Korrektheit von Standards zur Beschreibung der Wirklichkeit und der Berechtigung moralischer Handlungsnormen voneinander unterscheidbar sind. Eine Berufung auf eine integrativ-empirische Ethik verschleiert lediglich die Antwort auf die Frage, nach welchen Standards die Triftigkeit der entsprechenden Aussagen überprüft werden kann. Wie kann man ein theoretisches Hybrid kritisieren? Darf ich einem integrativen Ethiker vorwerfen, dass er die Praxis der Sterbehilfe nicht gut beschrieben hat? Darf ich ihn kritisieren, weil er die Meinung der Bevölkerung falsch wiedergegeben hat? Oder darf ich ihm vorwerfen, dass die Handlungsnorm, die er vertritt, nicht gut begründet ist? Wenn aber nicht ersichtlich ist, welchem wissenschaftlichen Status eine Hypbrid-Theorie verpflichtet ist, ist nicht ersichtlich, mit welchen Argumenten und unter Rückgriff auf welche Theorie-Kontexte legitimer Weise eine Kritik erfolgen kann. Insgesamt ist mir nicht deutlich, wie die ,integrative empirische Ethik" ihr Verhältnis zum allgemeinen ethischen Diskurs bestimmt. Entweder sie verteidigt die Triftigkeit dieses Ansatzes im Vergleich mit den verfügbaren metaethischen Alternativen, dann ist sie notwendig Teil des allgemeinen ethischen Diskurses und müsste sich darin beurteilen lassen. Oder sie versteht sich als Alternative zu diesem Diskurs, dann blendet sie einfach aus, dass Grundlagenfragen in der Ethik umstritten sind. Das Letztere wäre hingegen naiv oder arrogant, je nachdem ob dieses Problem überhaupt bewusst ist.

Ich würde dagegen vorschlagen, das Verhältnis zwischen verschiedenen Fragestellungen und Theorieformen grundsätzlich nach dem Muster gemischter Urteile zu konzipieren ([7], S. 5-10), was kurz ausgelegt werden soll: Als normative Disziplin geht es in der Medizinund Bioethik (wie in jeder Form angewandter Ethik) stets um Fragen nach dem richtigen Handeln. Die übergeordnete Frage lautet also: Wie sollen wir handeln? An unserem Beispiel: Wie sollen wir Fragen der Entscheidungen am Lebensende regeln? Zur Beantwortung dieser normativ-ethischen Fragestellung sind weitere Fragen unterschiedlicher Art zu beantworten. Ein Teil dieser Fragen richtet sich darauf, in Erfahrung zu bringen, wie eine Praxis derzeit faktisch aussieht. Ein Teil dieser Fragen richtet sich darauf zu ermitteln, welche Möglichkeiten der juridischen Regeln es überhaupt gibt und welche begründeten Einschätzungen wir haben um zu prognostizieren, wie sich bestimmte Regelungsformen auswirken werden. Diese Fragen sind also teils empirische, teils prognostische und teils konzeptuelle oder normative Fragen.

Eine ethische Studie kann also etwa zu dem Urteil kommen: „Aktive Sterbehilfe ist moralisch unzulässig“. Dieses Urteil kann dabei auf dem normativen Urteil beruhen: „Die selbstbestimmte Entscheidung des Sterbenden ist unbedingt zu achten" und auf dem empirischen Urteil: „In Krankenhäusern werden Entscheidungen über den Umgang mit Sterbenden gemäß der Willkür von Ärzten getroffen“, in Zusammenhang mit der prognostischen Einschätzung: „Eine Freigabe der aktiven Sterbehilfe wird die Willkür ärztlichen Handelns noch erweitern". (Die genannten Urteile sind bewusst schematisch und am Rande der Karikatur.) Das Endurteil (,Aktive Sterbehilfe ist moralisch unzulässig“) ist nun von der Validität aller genannten Teilurteile abhängig. Es handelt sich also um ein gemischtes Urteil, dessen Triftigkeit wiederum von der Berechtigung aller Teilurteile abhängt. Sofern sich eines der Teilurteile verändert, müsste das Endurteil geändert oder zumindest modifiziert werden: 
Wenn ich zum Ergebnis komme, dass die normative Voraussetzung nicht verteidigbar ist, so ändert sich das Endurteil ebenso wie durch eine Widerlegung der empirischen oder prognostischen Voraussetzungen. Dabei kann es auch möglich sein, dass sich bestimmte Voraussetzungen nicht begründen bzw. belegen lassen. In dem Fall müsste ein Urteil geformt werden, das der Tatsache Rechnung trägt, dass manche Fragen (noch) nicht entscheidbar sind.

Für die vorgeschlagene Konzeptualisierung gemischter Urteile wird hier keine Originalität beansprucht; vielleicht wird man in irgendeiner Form eine Linie zu Aristoteles' praktischem Syllogismus zeichnen können, aber das ist hier nicht wesentlich. Entscheidend ist vielmehr, dass die Berechtigung der genannten Teilurteile in unterschiedlichen Diskursen erwiesen werden muss, die keinen notwendigen methodischen und theoretischen Zusammenhang aufweisen. So hängt die Antwort auf die Frage, ob faktisch der Umgang mit Sterbenden von einer Willkür von Ärzten gekennzeichnet ist, in keiner Weise davon ab, ob Respekt vor der Selbstbestimmung von Patienten berechtigterweise gefordert werden kann, und umgekehrt. Es wird damit auch nicht behauptet, dass Fakten und Normen irgendwie ontologisch getrennt seien. Es wird lediglich gefordert, dass der Zusammenhang von der Begründung der Legitimität von Handlungsnormen und der zutreffenden Beschreibung der Praxis methodisch kontrolliert erfolgt. Dabei kommt in ethischen Untersuchungen (im Unterschied etwa zu soziologischen Studien) der unterstellten normativ-ethischen Theorie eine zweifache Rolle zu: Einerseits liefert sie gewissermaßen den normativen Ausgangspunkt, den Gesichtspunkt also, in dessen Licht die anderen Teilurteile normativ zu interpretieren sind. Andererseits bestimmt sich durch die normativ-ethische Theorie, welche empirischen und prognostischen Fragen zur Begründung des fraglichen moralischen Urteils überhaupt gestellt werden müssen. Dieser letzte Punkt ist alternativlos, wenn meine Argumentation stimmt, dass sich die Bedeutungen einiger empirischer Fragen nur aus der Perspektive bestimmter ethischer Theorien rekonstruieren und begründen lassen.

Die Konsequenz der bisherigen Überlegungen ist, dass ein Rückgriff auf empirische Untersuchungen nicht um die Schwierigkeiten der Pluralität von moralischen Überzeugungen und ethischen Konzeptualisierungen herumkommt. Das heißt, wenn erst einmal normativ begründet werden muss, welche empirischen Fragen zur Beantwortung moralischer Fragestellungen untersucht werden müssen, so kann auch eine empirisch arbeitende Bioethik den moralischen und ethischen Pluralismus nicht umgehen. Auch empirische Untersuchungen im Rahmen der Ethik werden in normativer Hinsicht Stellung nehmen müssen, um zu argumentieren, warum diese Untersuchungen ethisch relevant sind. Solch eine Stellungnahme wird aber stets normativ-ethische und metaethische Voraussetzungen machen, die ihrerseits umstritten sind. Angesichts der Tatsache, dass die Grundlagenfragen in der Ethik umstritten sind, gibt es keine Alternative dazu, dass jeder Ethiker seine eigenen normativen Voraussetzungen offenlegt und begründet.

\section{Interdisziplinarität}

Wenn die bisherigen Überlegungen zutreffen, so wird man das Verhältnis von empirischer und ethischer Forschung als eine interdisziplinäre Zusammenarbeit begreifen müssen. Diese Zusammenarbeit steht aber in diesem Fall vor einer Reihe von spezifischen Herausforderungen. Der erste Punkt, der besondere Aufmerksamkeit fordert, ist die Tatsache, dass Sozialwissenschaftler und Ethiker in mancher Hinsicht den gleichen Gegenstand haben: Normen menschlichen Handelns. Sie reflektieren diesen Gegenstand aber in unterschiedlicher Weise. Dabei gibt es eine Reihe von theoretischen Konzeptualisierungen, die strikt unverträglich miteinander sind. Auf zwei Unverträglichkeiten will ich kurz hinweisen: Da 
ist zunächst der Handlungsbegriff. Einige etwa systemtheoretische Konzeptionen (z.B. vom späten Luhmann [15]) versuchen den Handlungsbegriff zu vermeiden. Andere dagegen (etwa Latour [12]) verwenden einen nicht-intentionalen Handlungsbegriff, der den Unterschied zwischen Handlungen und Ereignissen tendenziell nivelliert. Ethik jedoch fragt nach der Berechtigung von Handlungsnormen. Das bedeutet, dass für die Frage, worauf ethische Theorien eine Antwort geben wollen, der Handlungsbegriff und alle damit verbundenen Begriffe wie Verantwortung von zentraler Bedeutung sind. Versucht man eine Ethik zu etablieren, die ohne den Handlungsbegriff auskommt, so wäre zunächst zu klären, wie eine solche Ethik sich zur gesamten Tradition ethischer Theoriebildung verhält. Für die interdisziplinäre Zusammenarbeit bedeutet dies, dass Rückgriffe auf sozialwissenschaftliche Theoriebildungen zunächst klären müssten, wie sich zentrale theoretische Voraussetzungen zueinander verhalten.

Das gleiche gilt für die Auffassung von Ethik als (präskriptiver) Disziplin. Voraussetzung jeder interdisziplinären Zusammenarbeit ist es, dass die beteiligten Disziplinen ihre Fragestellungen und Methoden in ein reflektiertes Verhältnis setzen. Die minimale Voraussetzung dafür ist, dass sich die beiden Disziplinen überhaupt als Disziplinen anerkennen. Nun werden die Sozialwissenschaften von Ethikern in aller Regel als Disziplin akzeptiert, umgekehrt gilt dies häufig nicht. Dafür gibt es Gründe, die u.a. mit der Entstehensgeschichte der Sozialwissenschaften (etwa Diskussionen um Wertfreiheit) zu tun haben. Für die Zusammenarbeit wäre dies aber vorgängig zu klären. Wenn Ethik eine Reflexion auf die Richtigkeit von Handlungsnormen (oder wie immer man die präskriptive Dimension theoretisch formulieren möchte) darstellt, so ist die minimale Voraussetzung für eine interdisziplinäre Zusammenarbeit, dass Sozialwissenschaftler dies für ein mögliches Profil einer Disziplin ansehen.

Eine weitere Frage im Zusammenhang mit der interdisziplinären Zusammenarbeit in diesem Kontext richtet sich auf das Verständnis von Politischer Philosophie, Gesellschaftstheorie und Sozialtheorie. Medizinethik reflektiert auf Handlungsnormen, die sich sowohl auf individueller als auch auf kollektiver Ebene abspielen. Die meisten umstrittenen Fragen in der Medizin- und Bioethik haben jedoch mit dem Handeln von und in Institutionen zu tun, häufig auch auf der Ebene politischer Institutionen. Das setzt aber einen Begriff von Gesellschaft, von Institutionen und vom Politischen voraus. Im Bereich der angewandten Ethik ist es jedoch einer der wesentlichen Schwachpunkt, dass diese Dimension selten explizit reflektiert wird. Das ist jedoch ein Reflexionsdefizit, das zu einer hohen Hypothek für die interdisziplinäre Zusammenarbeit in diesem Feld führt.

\section{Forschungsethik}

Abschließend soll noch kurz auf die ethische Dimension der empirischen Ethikforschung eingegangen werden. Insofern es dabei um Forschung geht, sollte diese Forschung ebenso ethisch beurteilt werden wie andere Forschung auch. Nun könnte man der Auffassung sein, es sei wenig wahrscheinlich, dass in dieser Forschung moralisch problematische Dinge geschehen, denn immerhin wird hier nicht mit gefährlichen Substanzen gearbeitet, werden keine Tiere getötet oder Embryonen verbraucht. Gleichwohl geht es bisweilen um Forschungsvorhaben, die für Probanden durchaus belastenden Charakter haben können, etwa wenn besonders vulnerable Patientengruppen in Interviews mit relativ intimen Fragen konfrontiert werden oder terminal Krebskranke Fragen zum Patiententestament beantworten sollen. Es gibt daher durchaus Gründe, nach der Verantwortbarkeit dieser Forschung zu 
fragen. Zudem sollte man erwarten können, dass Ethiker in Bezug auf die eigene Forschung besonders aufmerksam und skrupulös bezüglich Fragen der Verantwortbarkeit sind.

Nun gibt es hier zumindest drei Fragen, die beantwortet werden müssen: (a) Fragen nach der Relevanz und Notwendigkeit der Forschung, (b) Fragen nach der Verantwortbarkeit des Forschungsdesigns und (c) Fragen nach der Qualifikation der Forscher. Generell kann ein Proband erwarten, dass ein Forschungsvorhaben, an dem er mitwirkt, zum Fortgang der Forschung beiträgt, dass das Forschungsprojekt gemäß erwartbaren Forschungsstandards entwickelt wurde und dass der Forscher die erforderliche Qualifikation besitzt, eine solche Forschung durchzuführen. Im Einzelfall ist es natürlich möglich, dass etwa die Relevanz eines Forschungsvorhabens im Voraus schwer zu beurteilen ist oder dass über die geeigneten Forschungsstandards keine Einigkeit besteht. Man sollte also vorsichtig sein und nicht versuchen, strittige Fragen über Forschungsziele und -methoden durch moralische Beurteilungen dieser Forschungen entscheiden zu wollen. Doch wenn systematisch Fragen nach der Verantwortbarkeit der Forschung nicht gestellt werden, dann kann dies zu einem Vertrauensverlust in die Forschung führen.

Im Fall der empirisch arbeitenden Ethikforschung wird nun regelmäßig der Verdacht geäußert, die Forschung habe sich deshalb so stark entwickelt, weil von Medizinethikern an medizinischen Fakultäten erwartet werde, dass sie empirisch arbeiten, und weil auch nur Promotionsarbeiten mit empirischem Forschungsanteil akzeptiert würden. Ich bin nicht in der Lage, empirisch zu prüfen, ob dieser Verdacht zutrifft. Für das Vertrauen in die Forschung, aber auch aus grundlegenden moralischen Erwägungen wäre es jedoch bedenklich, wenn dieser Verdacht zuträfe. Die Forschung muss also die Frage beantworten können: Warum benötigt man das empirische Wissen, das erhoben wird, um ethische Fragen zu beantworten? Warum werden welche Forschungsmethoden gewählt? Und schließlich: Welche Qualifikation darf man von den betroffenen Forschern erwarten? Ich habe weiter oben gezeigt, dass zunächst in einer normativen Argumentation gezeigt werden müsste, welches spezifische empirische Wissen erforderlich ist, um eine spezifische normativ-ethische Frage zu beantworten. Dabei wurde auch gezeigt, dass es von den angenommenen jeweiligen normativen Ausgangspunkten abhängt, welches empirische Wissen genau erforderlich ist, was dann auch für die Wahl der entsprechenden sozialwissenschaftlichen Methodologie ausschlaggebend sein sollte. Sowohl über die Begründung der Notwendigkeit des entsprechenden empirischen Wissens als auch über die Wahl der geeigneten sozialwissenschaftlichen Methodologie kann man unterschiedliche Positionen vertreten. Aber man wird erwarten können, dass in empirischen Studien im Kontext der Medizinethik eine moralphilosophische Begründung für die Notwendigkeit der empirischen Studie zur Beantwortung moralischer Fragen und des entsprechenden Studiendesigns zu finden ist. Ferner aber wird man erwarten können, dass die Forschenden dafür qualifiziert sind; sicherlich werden die Probanden dies erwarten können. Nun müssen Qualifikationen nicht notwendig durch Formalqualifikationen belegt sein, wenngleich der Mangel solcher Qualifikationen zumindest Fragen aufwirft. Sicherlich fragwürdig ist es jedoch, wenn Studierende der Medizin im Rahmen medizinethischer Promotionen empirische Studien durchführen, denn ein Studium der Humanmedizin allein qualifiziert weder dazu, ethische Argumentationen nach Standards philosophisch-ethischer Theoriebildung durchzuführen, noch zu reflektierter Wahl geeigneter sozialwissenschaftlicher Forschungsmethoden, noch zu einer kundigen Durchführung und ethischen Interpretation der empirischen Studien. Wenn Ethik die Instanz sein soll, die über die moralische Vertretbarkeit und Verantwortbarkeit von Forschung nachdenkt, so sollte sie mit ihrer eigenen Forschung beginnen. 
Open Access Dieser Artikel unterliegt den Bedingungen der Creative Commons Attribution Noncommercial License. Dadurch sind die nichtkommerzielle Nutzung, Verteilung und Reproduktion erlaubt, sofern der/die Originalautor/en und die Quelle angegeben sind.

\section{Literatur}

1. Audi R (2004) The good in the right. A theory of intuition and intrinsic value. Princeton UP, Princeton

2. Birnbacher D (1999) Ethics and social science: Which kind of co-operation? Ethical Theory Moral Pract 2:319-336

3. Borry P, Schotsmans P, Dierickx K (2004) What is the role of empirical research in bioethical reflection and decision-making? An ethical analysis. Med Health Care Philos 7:43-53

4. Borry P, Schotsmans P, Dierickx K (2005) The birth of the empirical turn in bioethics. Bioeth 19(1):49-71

5. Brody BA (1993) Assessing empirical research in bioethics. Theo Med 14:211-219

6. Düwell M (2005) Sozialwissenschaften, Gesellschaftstheorie und Ethik. Jahr Wiss Ethik 10:5-22

7. Düwell M (2008) Bioethik. Methoden, Theorien und Bereiche. Metzler, Stuttgart

8. Häyry M, Takala T, Herissone-Kelly P (eds) (2005) Bioethics and social reality. Value inquiry book. Rodopy, Amsterdam

9. Holm S, Jonas MF (eds) (2004) Engaging the world: The use of empirical research in bioethics and the regulation of biotechnology. IOS Press, Amsterdam

10. Keulartz J, Schermer M, Korthals M, Swierstra T (2004) Ethics in technological culture: A programmatic proposal for a pragmatist approach. Sci, Technol Human Values 29(1):3-29

11. Krones T (2008) Kontextsensitive Bioethik - Wissenschaftstheorie und Medizin als Praxis. Campus, Frankfurt am Main

12. Latour B (2005) Reassembling the social: An introduction to actor-network-theory. Oxford UP, Oxford

13. Levitt M (2003) Public consultation in bioethics. What's the point of asking the public when they have neither scientific nor ethical expertise? Health Care Anal 11(1):15-25

14. Levitt M (2003) Better together? Sociological and philosophical perspectives on bioethics. In: Häyry M, Takala T (eds) Scratching the surface of bioethics, Rodopy, Amsterdam, pp 19-27

15. Luhmann N (1997) Die Gesellschaft der Gesellschaft, 2 Bde. Suhrkamp, Frankfurt am Main

16. Molewijk B, Stiggelbout AM, Otten W, Dupuis HM, Kievit J (2003) Implicit normativity in evidencebased medicine: A plea for integrated empirical ethics research. Health Care Anal 11(1):62-92

17. Molewijk B, Stiggelbout AM, Otten W, Dupuis HM, Kievit J (2004) Empirical data and moral theory. A plea for integrated empirical ethics. Med Health Care Philos 7:55-69

18. Molewijk B (2004) Integrated empirical ethics: In search for clarifying identities. Med Health Care and Philos 7:85-87

19. Musschenga AW (2004) Empirisch geïnformeerde ethiek. Inaugural lecture. Vrije Universiteit, Amsterdam

20. Musschenga AW (2005) Empirical ethics: Contextsensitivity or contextuality. J Med Philos 30(5):467-490

21. Nijsingh N, Düwell M (2009) Interdisciplinarity, applied ethics and social science. In: Sollie P, Düwell $\mathrm{M}$ (eds) Evaluating new technologies. Methodological problems for the ethical assessment of technological developments. Springer, Dordrecht, pp 79-92

22. Putnam H (2002) The collapse of the fact/value dichotomy and other essays. Harvard, Cambridge/Mass

23. Scarano N (2001) Moralische Überzeugungen. Mentis, Paderborn

24. Schaber P (1997) Moralischer Realismus. Alber, Freiburg/Breisgau München

25. Scheer L van der, Widdershoven G (2004) Integrated empirical ethics: Loss of normativity? Med Health Care Philos 7:71-79 